\title{
La ressource géothermique française Utilisation et perspectives
}

\author{
The french geothermal resource \\ Utilization and prospects
}

\author{
J.C. Vathaire
}

Directeur technique de GEOTHERMA

Suscitée en 1974 par la crise de l'énergie et intensifiée à partir de 1979, en Ile-de-France et en Aquitaine, l'exploitation de la ressource géothermique est en bonne voie: 60 forages réalisés, dont 40 en exploitation, qui permettent de chauffer plus de 600000 personnes,

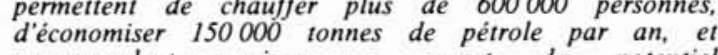
correspondent environ au quart du potentiel économiquement disponible.

Ce développement classe actuellement la France au $I^{\mathrm{c}}$ rang mondial pour l'utilisation de l'énergie géothermique basse température, et constitue une technologie dès à présent exportable (chiffre d'affaires actuel de plus d'un milliard de francs)
Spurred in 1974 by the energy crisis and intensified after 1979 in the Ile de France and Aquitaine regions, exploitation of the geothermal resource is progressing satisfactorily : 60 drill holes made, including 40 in exploitation, providing heating for more than 600,000 persons, allowing an annual savings of 150,000 metric tons of oil, and corresponding to approximately a quarter of the economically available potential. This development makes France the world leader for use of low temperature geothermal power and is already an exportable technology (current turnover more than one billion French francs).
En 1974, l'énergie géothermique est apparue aux yeux des géologues français comme une des formes d'énergie susceptible d'être rapidement utilisable pour pallier la première crise du pétrole.

L'utilisation thermique des sources chaudes remonte à la plus haute antiquité, et les premières applications industrielles sont apparues au début de ce siècle, en Hongrie pour la production de chaleur et en Italie pour la production d'électricité. En 1974, près de 40 pays recouraient à ce type d'énergie, essentiellement pour la production d'électricité, à partir de gites de vapeur à haute température.

La France métropolitaine est dépourvue de telles ressources, mais elle dispose de vastes bassins sédimentaires recélant des aquifères profonds, essentiellement connus grâce aux travaux de recherche pétrolière. Ainsi, la première expérience était tentée en 1964 au forage de Carrières-sur-Seine, au nord-ouest de Paris, par la C.E.P. (Compagnie d'Exploration Pétrolière), maintenant re- groupée au sein de ELF-ERAP. Mais la minéralisation importante des eaux du Dogger (de 10 à $15 \mathrm{~g} / \mathrm{l}$ ) avait alors découragé les futurs utilisateurs.

Le mérite de "l'invention " du dispositif d'exploitation de telles eaux, le doublet, revient à un ingénieur pétrolier, Pierre Maugis, qui, à la suite de l'expérience de Carrières, imaginera de réinjecter l'eau, permettant ainsi, après épuisement calorifique par échange, d'éviter le rejet en surface d'une eau fortement minéralisée, et de maintenir la pression du gisement. Cette conception devait aboutir à la réalisation, en 1969 à Melun l'Almont au sud-est de Paris, du premier doublet géothermique permettant d'assurer une part importante du chauffage et de la production d'eau chaude sanitaire de plus de 2500 logements collectifs, à partir d'eaux à basse température $\left(72^{\circ} \mathrm{C}\right)$ issues de la nappe du Dogger, à 1700 mètres de profondeur. Cette installation est maintenant en exploitation depuis 14 ans et permet d'économiser 1500 TEP (tonnes d'équivalent-pétrole) par an. 
S'appuyant sur cette première expérience et sur les données acquises par les géologues grâce aux travaux de recherche pétrolière, le gouvernement français décidait, dès 1974, d'utiliser l'énergie géothermique de nos aquifères profonds. L'objectif fixé début 1975 était de 500 doublets à réaliser d'ici 1985 pour assurer le chauffage d'un million de logements. Fin 1984, 50 installations sont en exploitation, économisant annuellement 180000 TEP. Une vingtaine d'autres sont en cours d'équipement ou de réalisation.

\section{$*$}

Un certain nombre d'obstacles ont entravé le développement de cette énergie, les uns économiques, les autres techniques.

Obstacles économiques, car le coût d'investissement initial reste élevé (de 40 à 50 millions de Francs) par rapport à la mise en œuvre d'autres énergies. Un tel investissement, qui comporte une préférence du long terme (économies de combustible) sur le court terme (investissements) est fortement dépendant des taux d'intérêt ( $14 \%$ en moyenne) et du prix des énergies de référence (sous-évalué pour certaines) Or, à la différence des secteurs traditionnels de l'énergie, en géothermie les investissements de production et de distribution sont à la charge directe des décideurs (en général collectivités locales). Si un tel dispositif est particulièrement avantageux pour l'usager, il pèse particulièrement lourd dans les programmes d'investissements des collectivités locales.

Et l'on voit ainsi trois étapes dans le développement de cette énergie en France :

— de 1974 à 1979 : 5 opérations seulement seront réalisées alors que le coût du pétrole en Francs constants, après une brusque hausse en 1974, redevient stable, sinon régresse,

— de 1979 à 1983 : 35 opérations engagées, réalisées et mises en exploitation, après le deuxième choc pétrolier et l'envolée des prix,

- en 1983 et 1984 : ralentissement de l'activité face à une relative surabondance énergétique, à une régression des coûts de l'énergie et aux difficultés de financement des collectivités locales.

Obstacles techniques, car deux contraintes conditionnent essentiellement l'utilisation de cette énergie. La première est due au fait que le marché de la chaleur géothermique est un marché captif. Celle-ci ne peut être utilisée que sur place, à la différence des ressources minières classiques. La faisabilité économique d'une exploitation géothermique dépendra donc à la fois des caractéristiques de la ressource (débit, température) et de celles de l'habitat consommateur. Ce sont généralement ces dernières qui sont le plus critiques (densité et quantité des logements raccordés, mode de chauffage ou type d'émetteur, type d'énergie substituée).

Les grands inventaires entrepris dès 1975 (Bassin Parisien, Bassin Aquitain, Alsace) ne s'intéressaient primitivement qu'à la ressource. Rapidement, on dut y adjoindre un recensement détaillé des utilisateurs (inventaires départementaux).

La deuxième contrainte est apparue au cours des explorations menées dans les différents bassins sédimentaires français: l'existence d'une ressource géothermique basse température suppose des conditions hydrogéologiques très favorables (formations à très forte perméabilité : fissurées, fracturées karstifiées, ou à perméabilité moyenne mais épaisseur productive importante) compte-tenu des débits importants recherchés $\left(150\right.$ à $\left.300 \mathrm{~m}^{3} / \mathrm{h}\right)$. De plus, il est apparu des difficultés techniques provisoirement insurmontables lors de la réinjection de débits importants (supérieurs à $100 \mathrm{~m}^{3} / \mathrm{h}$ ) dans les formations détritiques, sableuses ou gréseuses (Trias région parisienne, Orléanais). Actuellement, ne sont exploités en doublet (avec réinjection) que les réservoirs calcaires, et en puits unique (sans réinjection) des formations calcaires et des formations sableuses ou gréseuses. Les ressources géothermiques apparaissent maintenant plus localisées qu'on ne le supposait au départ. Elles constituent à ce titre de véritables gisements miniers.

\section{$\therefore$}

Comment se répartissent ces gisements sur le territoire français? Essentiellement dans les deux plus grands bassins sédimentaires: le Bassin Parisien et le Bassin Aquitain.

Dans le Bassin Parisien, l'essentiel des opérations exploitent les calcaires du Dogger. Cette formation, remarquablement régulière, constitue un réservoir particulièrement favorable à l'exploitation goéthermique : la température des eaux varie en région parisienne de 55 à $70^{\circ} \mathrm{C}$ et atteint $85^{\circ} \mathrm{C}$ dans la zone la plus profonde du Bassin, pour des profondeurs variant de 1400 à 1900 mètres. Les débits peuvent être très importants (supérieurs à $200 \mathrm{~m}^{3} / \mathrm{h}$ ) dans toute la partie centrale du Bassin, axée sur la Brie, sur une superficie de plus de $9000 \mathrm{~km}^{2}$. Ils sont encore importants (supérieurs à $120 \mathrm{~m}^{3} / \mathrm{h}$ ) sur près de $8000 \mathrm{~km}^{2}$. A la périphérie, la productivité est nettement moins importante, et la température plus faible en raison de la profondeur moindre de cette formation.

L'eau contenue dans ces calcaires est salée, la salinité variant de 5 à $30 \mathrm{~g} / \mathrm{l}$, les eaux les plus minéralisées se situant au centre et au nord-ouest du bassin.

L'essentiel de l'agglomération parisienne et de sa couronne est situé à l'aplomb de cette ressource, ainsi qu'un certain nombre de villes importantes telles que Creil, Meaux, Melun ou Fontainebleau. Actuellement, 41 opérations sont en exploitation ou sur le point d'être exploitées sur cet aquifère, représentant plus de 125000 équivalents-logements raccordés et plus de 120000 TEP économisées.

Outre le Dogger, d'autres aquifères sont exploités par quelques opérations. En particulier, la partie inférieure du Crétacé comporte deux importants niveaux sableux, l'Albien et le Néocomien, le premier étant exploité pour l'alimentation en eau potable en région parisienne depuis 1941. De nombreux ouvrages exploitent cette nappe, parfois à des débits supérieurs à $200 \mathrm{~m}^{3} / \mathrm{h}$. Le seul puits réalisé à des fins géothermiques est celui destiné au chauffage de la Maison de la Radio (1960). Des sables de l'Albien sont présents sur une vaste étendue au centre du Bassin Parisien, la température des eaux oscillant entre 20 et $30^{\circ} \mathrm{C}$ suivant la profondeur de la nappe. Depuis 1935 , cette nappe est protégée afin d'éviter sa surexploitation.

Situés 150 mètres au-dessous de l'Albien, les sables du Néocomien constituent un objectif important sur une grande étendue du Bassin Parisien, la température des 


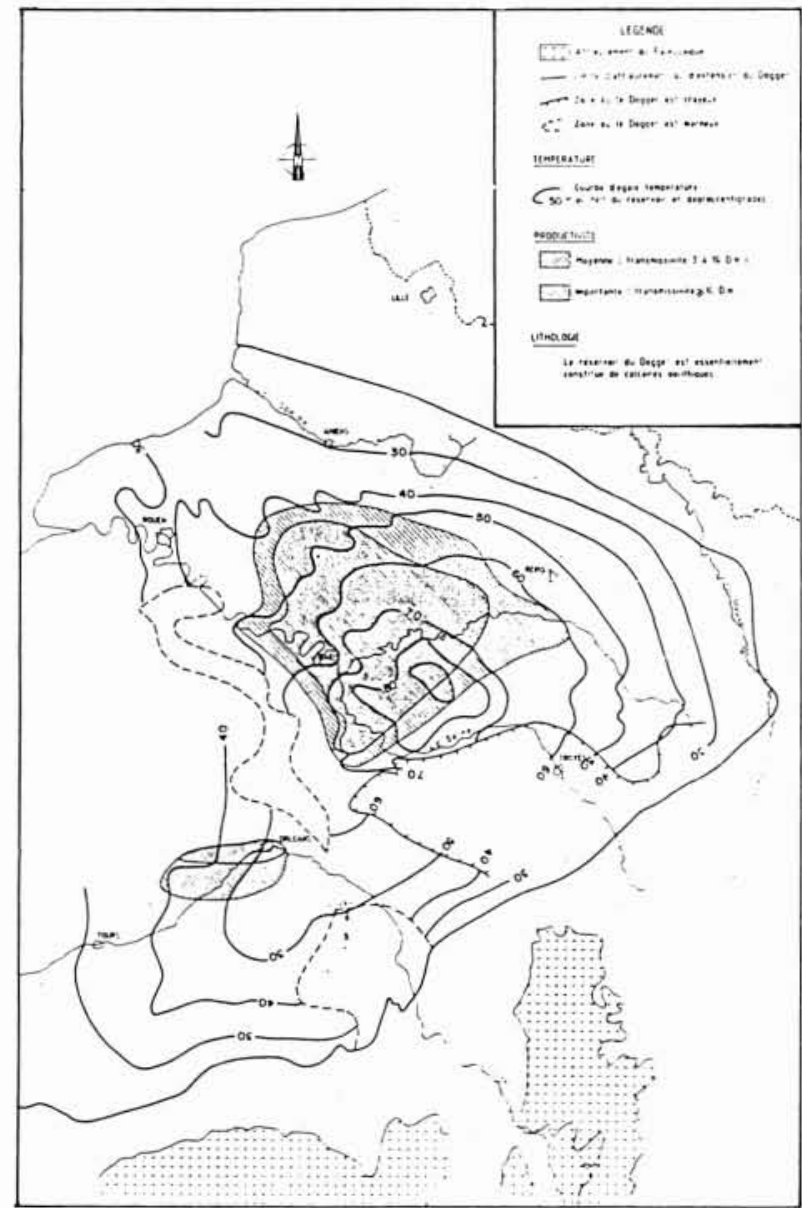

AQUIFÈRE GÉOTHERMIQUE DU DOGGER DU BASSIN PARISIEN

TEMPÉRATURE ET PRODUCTIVITÉ
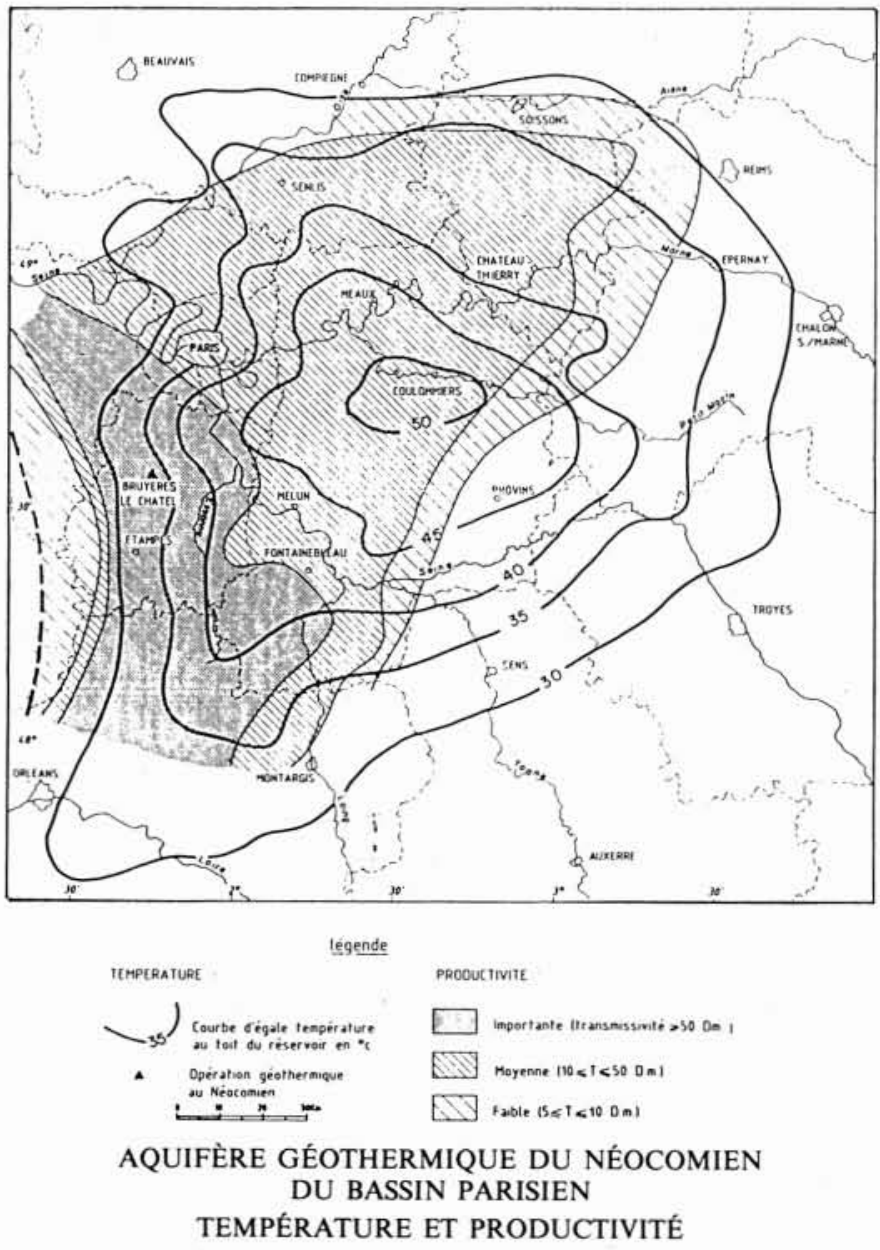

Réservoir le plus profond du Bassin Parisien, les formations détritiques du Trias constituent des objectifs potentiels en plusieurs régions du Bassin Parisien. Il est aussi le principal objectif en région Centre. Il va ainsi être exploité à Châteauroux où ces formations, à une profondeur de 700 mètres, fournissent une eau à plus de $30^{\circ} \mathrm{C}$. Ces formations s'ennoient rapidement vers le nord, la température des eaux contenues pouvant dépasser $80^{\circ} \mathrm{C}$ du cœur de la fosse de Sologne.

Autre région potentiellement intéressante pour l'exploitation du Trias, la Lorraine ne comporte pas encore d'exploitation à des fins géothermales.

En région parisienne, les formations détritiques du Trias peuvent constituer un objectif géothermique le long d'un axe centré sur la Basse Seine. Deux opérations ont jusqu'à présent tenté d'exploiter ces niveaux : la première à Cergy, la seconde à Achères. Dans les deux cas, il a été décidé de recourir à l'exploitation du Dogger après qu'il ait été constaté à Cergy que la productivité du réservoir était insuffisante, et à Achères que la réinjectivité était trop faible. La difficulté est analogue à celle rencontrée dans l'opération de Melleray (Saint-Denis-en-Val) près d'Orléans. 


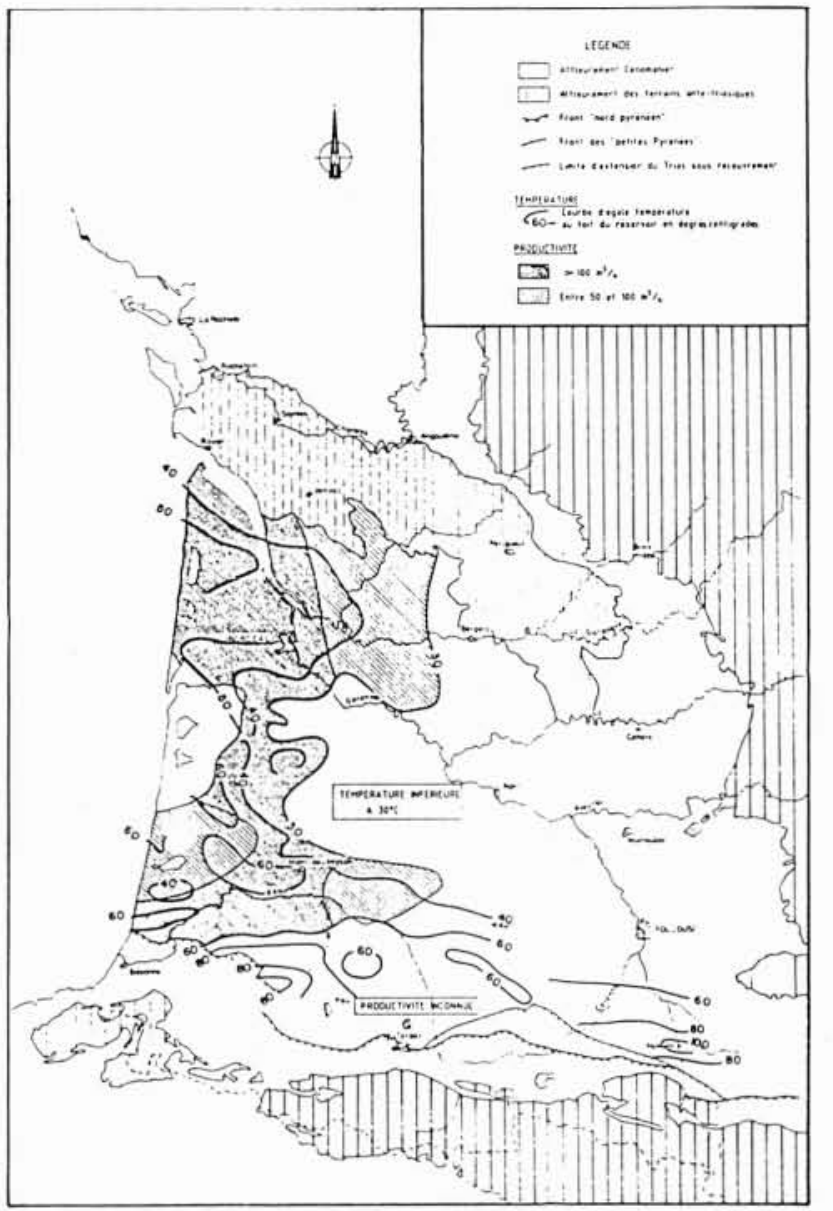

RÉSERVOIR GÉOTHERMIQUE

DU CENOMANO-TURONIEN DU BASSIN AQUITAIN TEMPÉRATURE ET PRODUCTIVITÉ

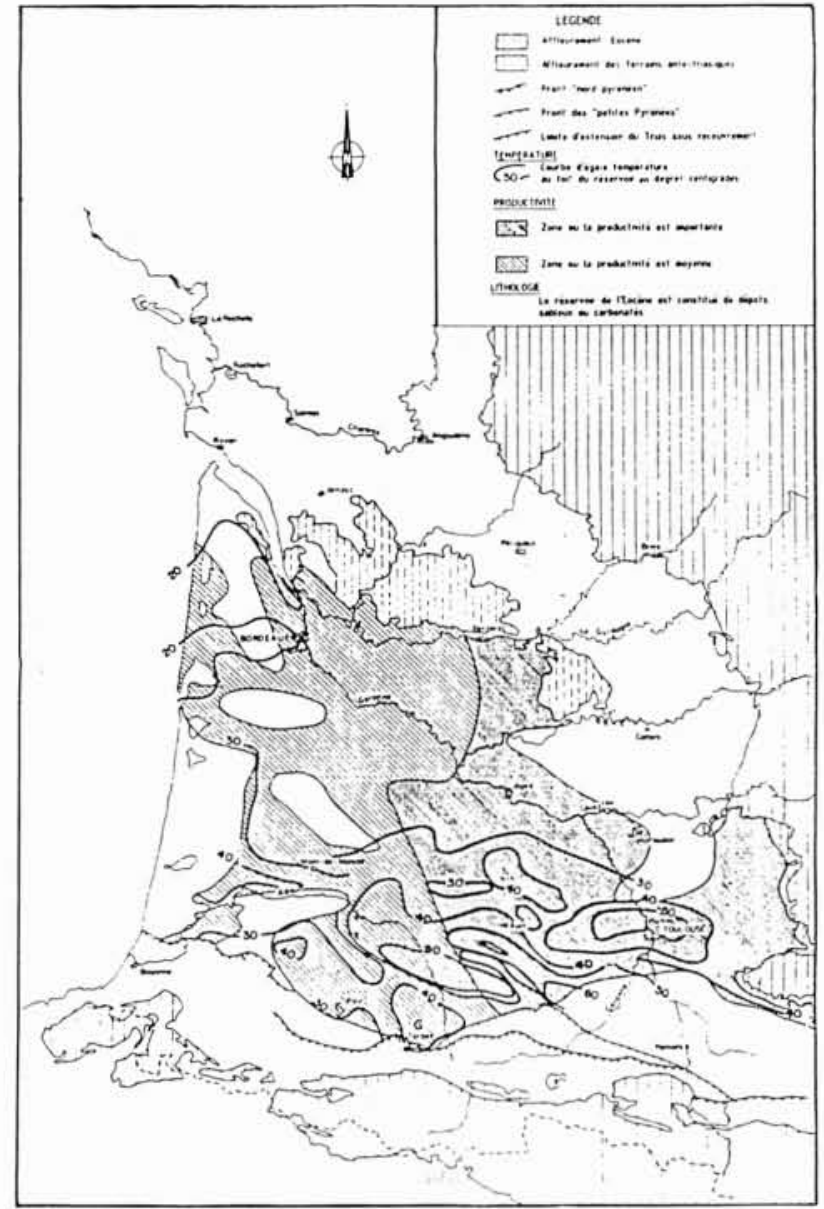

RÉSERVOIR GÉOTHERMIQUE

DE L'EOCENE DU BASSIN AQUITAIN TEMPÉRATURE ET PRODUCTIVITÉ
Compte tenu de ces difficultés, l'Agence Française pour la Maîtrise de l'Energie a décidé, courant 1983, de ne plus autoriser la réalisation de doublet au Trias avant que les problèmes d'injection ne soient résolus. En raison de l'importance du potentiel géothermique que représente le Trias pour la région parisienne, un important programme de recherches a été engagé par l'Institut Mixte de Recherches Géothermiques afin de déterminer les causes et les solutions à ces problèmes d'injection.

Les opérations déjà réalisées dans le Bassin Parisien se répartissent en 41 doublets au Dogger et 6 puits uniques (Albien, Néocomien, Lusitanien et Trias), soit au total 88 forages.

Le Bassin Aquitain constitue un bassin beaucoup plus complexe que le Bassin Parisien, à la fois par la variété de ses faciès et par sa structure tectonique profonde. Son sous-sol est riche en formation très perméables, karstifiées ou très fracturées. Les circulations y sont donc rapides, les eaux peu minéralisées et, de ce fait, n'exigent pas de forage de réinjection.

L'essentiel des opérations exploitent les eaux contenues dans les formations du Crétacé supérieur (Cénomanien, Turonien). Dans les Landes, les forages de Dax et de Mont-de-Marsan exploitent les eaux des dolomies karstiques. Si ce type de formation est susceptible de produire des débits importants (supérieurs à $200 \mathrm{~m}^{3} / \mathrm{h}$ ), par contre leur mise en production n'est pas sans risque compte-tenu du caractère bien souvent localisé des phénomènes de karstification (difficultés de mise en production à Mont-de-Marsan II). En région bordelaise, les forages réalisés (Mériadeck, Bénauge, Pessac) exploitent les eaux des réservoirs gréseux et carbonatés du Cénomanien et de la base du Turonien. Depuis le nord de la région bordelaise jusqu'au sud des Landes, les réservoirs du Crétacé supérieur constituent un des principaux objectifs du Bassin Aquitain.

Une des autres formations aquifères essentielles pour la géothermie est constituée par les dépôts sableux et carbonatés de l'Eocène. Déjà exploitées par de nombreux captages pour l'eau potable, les eaux de ces formations sont exploitées pour la géothermie soit par des réalisations nouvelles (Toulouse-Blagnac, Lamazère), soit en profitant de captage anciens (Nogaro, etc.). Les formations de l'Eocène recouvrent pratiquement toute la superficie du Bassin Aquitain. Leur profondeur, peu importante dans le nord du Bassin, dépasse 1000 mètres dans les parties sud et sud-est du Bassin, la température des eaux pouvant dépasser $50^{\circ} \mathrm{C}$. Malgré leur température peu élevée, les formations de l'Eocène constituent un objectif géothermique important étant donné leur faible minéralisation et l'excellente connaissance que les géologues en ont. 
Autre aquifère exploité pour la géothermie, à Jonzac, les grès du Trias qui peuvent constituer un objectif intéressant dans la partie nord de l'Aquitaine, avec des températures oscillant entre 50 et $80^{\circ} \mathrm{C}$.

La série sédimentaire du Bassin Aquitain contient d'autres niveaux aquifères potentiellement intéressants pour l'exploitation géothermique, en particulier :

- les formations carbonatées ou détritiques du Paléocène et du Danien, localisées dans le sud du bassin, avec des températures pouvant atteindre $80^{\circ} \mathrm{C}$,

- les formations du Crétacé inférieur, carbonatées ou détritiques, exploitables à des températures supérieures à $80^{\circ} \mathrm{C}$, dans les bassins de Parentis, de l'Adour et dans le sud du bassin,

- les formations du Jurassique supérieur à moyen, exclusivement carbonatées et constituées essentiellement, dans les bassins de Parentis et de l'Adour, par la dolomie de Mano (Portlandien, Kimméridgien supérieur) avec des profondeurs généralement supérieures à 1000 mètres, et, dans le secteur s'étendant d'Angoulème à Auch, par des calcaires (Kimméridgien, Dogger).

Ces formations, qui recèlent sans doute un important potentiel géothermique, sont encore mal connues et difficiles à évaluer.

D'autres bassins sédimentaires ont fait l'objet de recherches, d'inventaires, voire d'explorations jusqu'ici infructueuses.

Le premier étudié, après les bassins parisien et aquitain, a été l'Alsace, ou plus exactement le fossé rhénan, correspondant à un effondrement entre Vosges et Forêt Noire où l'on trouve, sous un épais remplissage de tertiaire et de quaternaire, une série sédimentaire analogue à celle du Bassin Parisien, du Trias au Jurassique. Si le gradient y est élevé $\left(4^{\circ} \mathrm{C} / 100 \mathrm{~m}\right.$ en moyenne), voire localement très élevé $\left(10^{\circ} \mathrm{C} / 100 \mathrm{~m}\right)$, en contrepartie il y règne une tectonique intense compartimentant les aquifères potentiels et rendant leur localisation délicate.

Dans la partie nord, de Colmar à Strasbourg, deux formations peuvent constituer des objectifs potentiels. En premier lieu, la " grande oolithe ", comparable au Dogger du Bassin Parisien; si la température des eaux peut dépasser $100^{\circ} \mathrm{C}$, par contre les productivités observées sont inférieures à celles obtenues dans le Bassin Parisien. En second lieu, les formations détritiques du Trias inférieur (Buntsandstein). Ce dernier aquifère était l'objectif du forage de Cronenbourg, dans la banlieue de Strasbourg, qui constitue jusqu’à présent le seul forage géothermique réalisé, du côté français, dans le fossé rhénan. $\mathrm{Ce}$ forage, qui a atteint 3400 mètres de profondeur, a mis en évidence des eaux dont la température dépassait $140^{\circ} \mathrm{C}$. Malheureusement, la productivité est restée très insuffisante.

Dans la partie sud (région de Mulhouse, Bâle), les calcaires du Jurassique supérieur peuvent constituer un objectif géothermique. Une opération est d'ailleurs envisagée à Mulhouse.

Autre fossé d'effondrement, la Limagne a fait très tôt l'objet d'une exploration basée sur des campagnes de recherches pétrolières antérieures. Si le gradient y est important $\left(100^{\circ} \mathrm{C}\right.$ à 1500 mètres de profondeur), les formations constituant l'objectif (grès du Stampien) pré-

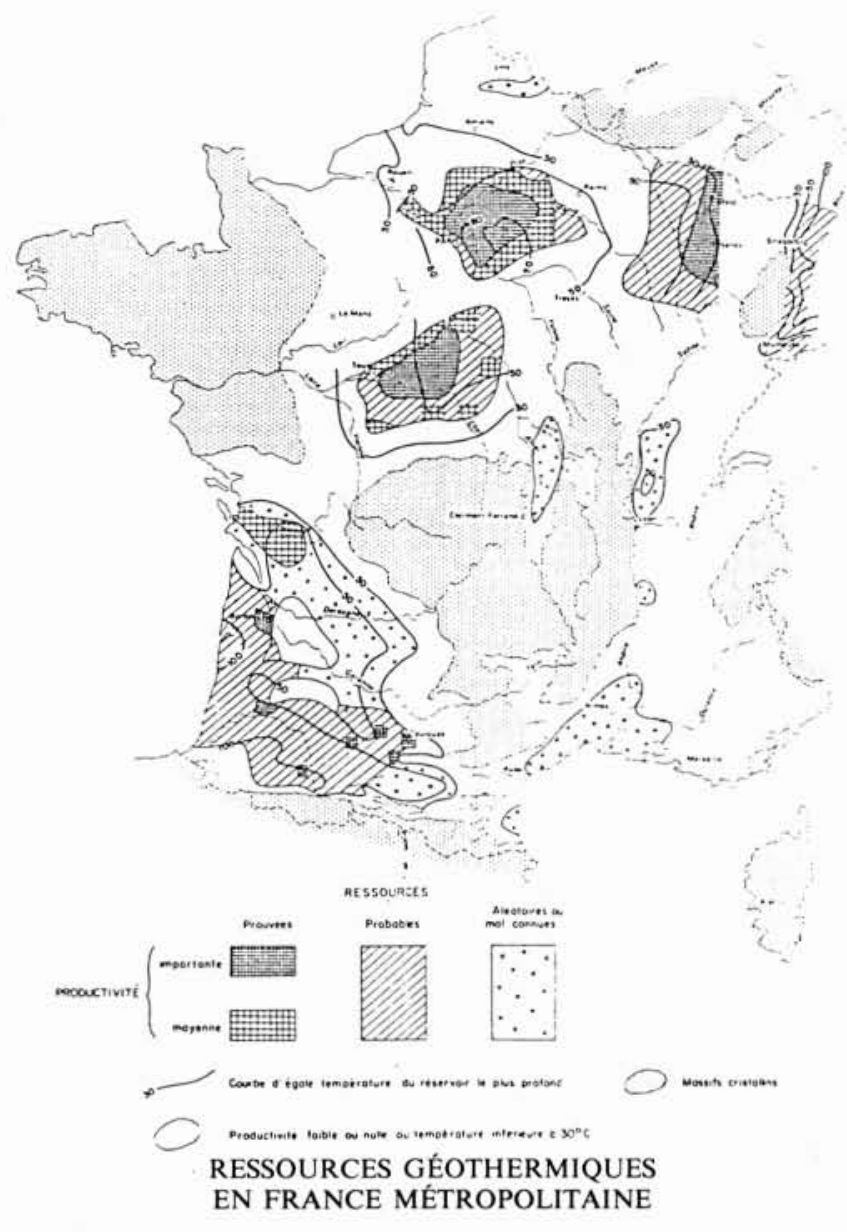

LOCALISATION ET DÉFINITION

DES RÉSERVOIRS GÉOTHERMIQUES DE LA FRANCE

\begin{tabular}{|c|c|c|c|}
\hline BASSIN DE PARIS & BASSIN AQUITAIN & ALSACE & AUTRES RÉGIONS \\
\hline 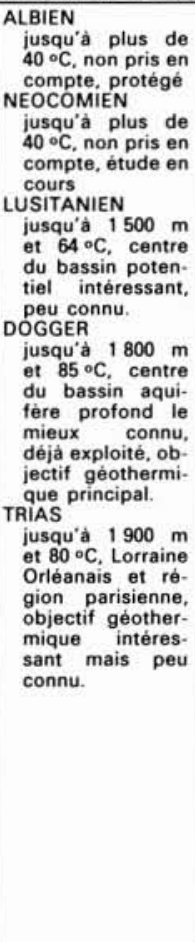 & 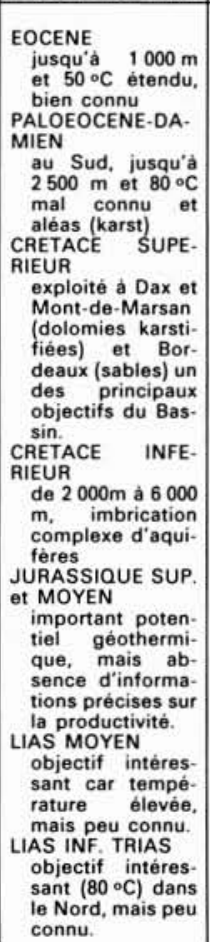 & 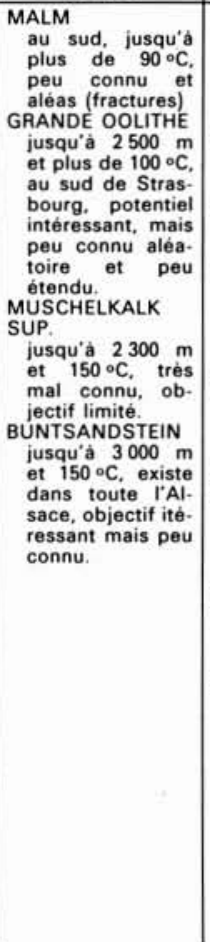 & 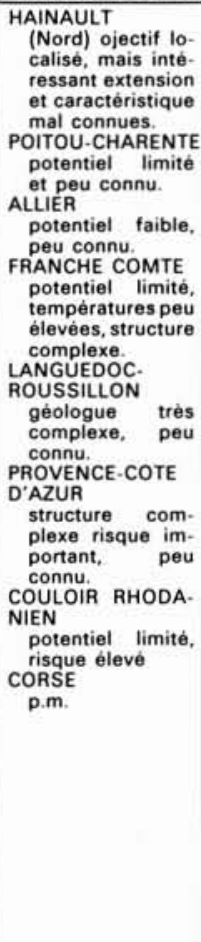 \\
\hline
\end{tabular}


sentent une productivité insuffisante. Deux forages y ont été réalisés: Beaumont, dans la banlieue sud de Clermont-Ferrand, les Vergnes, en banlieue nord. Ce dernier puits est actuellement l'objet de mesures complémentaires.

Autre zone d'effondrement également, mais beaucoup plus complexe que les deux précédentes, le couloir rhodanien, qui a subi fortement les effets de la tectonique alpine durant le tertiaire. Trois forages d'exploration y ont été menés sur des formations susceptibles de constituer des réservoirs d'extension régionale appartenant au Jurassique moyen et supérieur. Il s'agit là de calcaires où la ressource géothermale est localisée en milieu fissuré karstique, pouvant constituer des réservoirs importants, mais où le risque d'échec à l'exploration est important.

A des degrés divers, les forages réalisés à Bourg-enBresse, Valence et Avignon n'ont pas permis de mettre en évidence des réseaux karstifiés suffisamment importants, les températures observées correspondant généralement aux prévisions.

Localement, d'autres formations pourraient constituer des objectifs, mais elles n'ont généralement qu'une faible extension et présentent un risque élevé à l'exploration.

Le Languedoc-Roussillon a également été l'objet d'un inventaire détaillé. Sa complexité structurale, l'absence de continuité des réservoirs rendent particulièrement difficile l'exploitation de cette région. Plusieurs forages y ont déjà été réalisés, mais aucun d'entre eux n'a pu être mis en exploitation.

Dans le secteur de Montpellier ( $30^{\circ} \mathrm{C}, 500$ mètres), les calcaires du Dogger ont fait l'objet de deux forages (Antigone, Bagatelle), mais la ressource s'est avérée insuffisante.

Dans le secteur de Sète, les calcaires karstiques du Jurassique supérieur ont été explorés. Il en est de même à Agde, où le forage entrepris n'a pu atteindre cet objectif à la profondeur escomptée.

Un certain nombre d'objectifs à Béziers, Perpignan, Carcassonne, et aussi en Camargue et autour de l'Etang de Berre, peuvent constituer des réservoirs potentiels mais qui, compte-tenu de la faible connaissance que l'on en a, nécessiteront une stratégie d'exploration adéquate.

La dernière région qui n'a fait l'objet d'aucune exploration à ce jour, du côté français, est la région Nord/Pas-de-Calais. Il y a là un réservoir aquifère dans les calcaires du Carbonifère, le long de la bordure nord du bassin houiller franco-belge, dont l'existence a été prouvée par deux forages géothermiques réalisés en territoire belge et qui ont fourni des débits artésiens supérieurs à $100 \mathrm{~m}^{3} / \mathrm{h}$ à une température de l'ordre de $70^{\circ} \mathrm{C}$. La prolongation de ce réservoir en territoire français peut être supposée. Son évaluation nécessiterait des travaux complémentaires.

\section{*.}

Au total, à mi 1984 , ce sont près de 110 forages géothermiques qui ont été réalisés, dont :

$-80 \%$ en Bassin Parisien avec un taux de réussite de $96 \%$,

- $10 \%$ en Bassin Aquitain avec un taux de réussite de $75 \%$,
- $10 \%$ dans les autres bassins, sans résultat à ce jour.

Le secteur résidentiel, gros consommateur d'énergie, est à l'heure actuelle le secteur d'application de loin le plus important de la géothermie basse température. Les gammes de température utilisées sont très comparables à celles des ressources, les concentrations de logements sont à l'échelle des investissements nécessaires.

Le secteur tertiaire (équipements collectifs, sportifs, commerciaux, etc.) permet généralement l'utilisation optimale de la ressource géothermique.

Sur les 110 forages réalisés, il est frappant de constater que deux seulement, Melleray/Saint-Denis-en-Val et Lamazère, ont été réalisés pour le chauffage de serres, aucun pour le secteur industriel.

Ceci est révélateur des contraintes inhérentes à ce type d'énergie :

- conjoncture peu propice aux investissements lourds dont la rentabilité ne s'inscrit qu'à moyen et long terme; - concurrence vive des procédés d'économie d'énergie; - concurrence tout aussi importante des énergies traditionnelles (charbon, gaz, ou encore électricité) compte tenu des politiques tarifaires et de raccordement pratiquées, voire des sous-évaluations latentes (cf. déficits d'EDF et de GDF).

Dans la conjoncture actuelle, investir des sommes importantes pour utiliser ce type de ressources est-il justifié ?

Comme toute technologie nouvelle, la géothermie a connu une phase expérimentale de lancement, faite non seulement d'explorations infructueuses comme nous l'avons vu précédemment, mais aussi d'inadaptations, de sous-évaluations des investissements de surface, des consommations recensées, ou encore des frais financiers intercalaires. Les évaluations économiques récentes menées par l'Agence Française pour la Maîtrise de l'Energie montrent que la phase expérimentale de lancement est aujourd'hui dépassée et que les opérations sont réalisées dans des conditions économiques très intéressantes pour la majorité des promoteurs et pour les Pouvoirs Publics. En effet, le taux de rentabilité interne avant subvention est toujours supérieur à $9 \%$ pour une durée de vie des installations de 20 ans. Le calcul du taux de rentabilité prend en compte dans ce contexte la construction de réseaux de chaleur. Si ceux-ci existaient déjà, la compétitivité des MWh géothermiques serait extraordinaire.

Quelle est la constitution de l'investissement en géothermie ? En moyenne $38 \%$ de cet investissement seront consacrés au sous-sol, $54 \%$ à la surface, $8 \%$ à des investissements divers. Sur 18 opérations récemment lancées, le montant total de l'investissement varie entre $37000 \mathrm{kF} \mathrm{HT}$ et $128000 \mathrm{kF}$ HT suivant l'importance des réseaux et le nombre de doublets, soit une moyenne de l'ordre de $51300 \mathrm{kF} \mathrm{HT}$ par opération, et un montant de 11600 F HT par TEP substituée. Le taux de rentabilité interne de ces opérations varie de 9 à 14,93 (moyenne 12,14 ), le temps de retour de 5,2 ans à 8,9 ans (moyenne 7,5 ans). L'économie réalisée la première année d'exploitation varie de $2,1 \%$ à $18,8 \%$ (moyenne $8,57 \%$ ).

Les 18 opérations ainsi analysées sont celles de Garges-les-Gonesse, Tremblay-les-Gonesse, Bondy-Nord, Aulnay Gros Saule, Epinay-sous-Sénart, Cachan $\left(1^{\text {er }}\right.$ doublet), Sucy-en-Brie, Maisons-Alfort Sud, Villiers-le- 
Bel/Gonesse, Chevilly-Larue/L'Hay-les-Roses (2 doublets), Soisy/Eaubonne, Bagneux, Champigny, Chelles, Thiais, Bonneuil, Orly II, Créteil, correspondant à des opérations dont les débuts de travaux vont du $2^{c}$ semestre $1983{\mathrm{au} 1^{\text {er }}}$ semestre 1985.

L'ensemble de ces 18 opérations représente 83623 logements, soit une moyenne de 4400 logements par doublet, et un investissement moyen de $11650 \mathrm{~F} \mathrm{HT}$ par logement.

Comment se situe le coût de l'énergie géothermique par rapport aux énergies classiques des réseaux de chaleur? Une étude récente (septembre 1984), menée par la Fédération Nationale des Collectivités Concédantes et Régies (FNCCR) et le CNET-HLM à la demande de l'AFME, donne l'ordre suivant :

- la géothermie en-dessous de $200 \mathrm{~F}$ HT par MWh,

- le charbon de 140 à $240 \mathrm{~F} \mathrm{HT}$ par MWh,

- le fuel lourd de 180 à $320 \mathrm{~F}$ HT par MWh.

Encore cette étude n'est-elle menée que sur des opérations relativement anciennes. Les opérations précédemment citées devraient aboutir à un coût de l'énergie géothermique encore nettement plus compétitif.

Ce facteur est particulièrement important, d'autant que la géothermie peut être considérée comme une énergie véritablement nationale, une statistique récemment effectuée montrant en effet que la part de fournitures et services étrangers dans une opération de géothermie ne représente que $3,5 \%$ de l'investissement total en moyenne, alors que la part du fuel et des autres énergies payables en devises (charbon, gaz) reste encore très importante dans le chauffage et la production d'eau chaude sanitaire pour les secteurs résidentiels et tertiaires.

Enfin, il faut noter que si les investissements concernant la surface n'ont pas augmenté, par contre ceux concernant le sous-sol ont connu, entre fin 1980 et fin 1983, une baisse d'environ $13 \%$ de leur valeur en francs constants, avec deux grandes progressions technologiques :

- une diminution très importante des durées de forage (100 à 110 jours fin 1980 à 60 jours fin 1983, par doublet), - une amélioration des équipements, en particulier concernant les pompes d'exhaure.

Il est encore trop tôt pour réaliser une évaluation précise du coût d'exploitation de ces installations. Seules quelques-unes fonctionnent depuis quelques années :

- Melun d'Almont depuis 1971,

- Villeneuve-la-Garenne depuis 1976,

- Creil depuis 1976,

- Le Mée-sur-Seine depuis 1978,

- Coulommiers depuis 1982.

Toutes les autres opérations ont vu leur mise en service s'étager entre 1982 et 1984 . D'ores et déjà, des progrès substantiels ont été faits quant à la fiabilité des installations.

Ceci n'a pu se faire que grâce à la structuration d'un secteur industriel nouveau. Rappelons qu'en 1975, les moyens d'atteindre les objectifs envisagés puis fixés au $I^{c}$ plan n'existaient pas. Pour répondre à la demande des Pouvoirs Publics, les industriels français ont embauché et investi.
De 1979 à 1981, le parc de machines de forage consacrées à la recherche géothermique passait de 2 à 6 appareils, représentant un investissement de 140 millions de francs. Plus de 500 personnes étaient embauchées, des sociétés se créaient, particulièrement en matière d'ingéniérie de sous-sol et d'ingéniérie thermique spécialisées.

Début 1984 , le potentiel industriel de la géothermie française est constitué, entre autres, par :

- 6 machines de forage spécialisées et insonorisées afin de pouvoir travailler en zone urbaine à 2000 mètres de profondeur et plus de $45^{\circ}$ de déviation,

- 4 sociétés d'ingéniérie de sous-sol spécialisées dans la recherche et la mise en production de l'énergie géothermique basse et haute température,

- plus de 10 sociétés d'ingéniérie thermique spécialisées dans l'utilisation de la géothermie,

- près de 8 sociétés spécialisées intéressées par la production de tubes pour les forages ou pour les canalisations de surface,

- plus de 20 sociétés qui se sont spécialisées dans la pose des réseaux de chaleur et dans le génie climatique,

- plus de 10 sociétés qui ont investi dans la recherche et l'innovation pour la fabrication d'échangeurs à plaques ou de pompes à chaleur.

L'ensemble de ces moyens représente actuellement près de 3500 personnes dont l'activité est consacrée à la mise en exploitation et à l'utilisation de cette énergie, représentant un chiffre d'affaires annuel d'environ 1 milliard de Francs.

L'industrie française de la géothermie a actuellement un potentiel qui n'est utilisé qu'à $25 \%$ de sa capacité. En effet, la baisse du nombre d'opérations engagées en 1983 et la confirmation de cette tendance en 1984 n'engage pas à l'optimisme.

\section{$*$}

Quelles sont les perspectives ? Face à une conjoncture énergétique fluctuante et à un cours du dollar non moins hésitant, le développement de l'utilisation de tels types de ressources passe par l'abaissement des investissements initiaux.

Le bon moyen est l'exploitation des ressources à faible et moyenne profondeur.

Le gisement géothermique moyenne profondeur est évalué à 600000 TEP, à comparer aux $1586 \mathrm{M}$ TEP du potentiel géothermie profonde. Pour l'Ile-de-France, qui est de loin la première région pour le développement géothermique, le potentiel profond (Paris non compris) Trias + Dogger est de $1130 \mathrm{kTEP}$, tandis que le potentiel moyenne profondeur (Lusitanien + Néocomien) est de $490 \mathrm{kTEP}$, soit $43 \%$ environ du seul potentiel profond, et $30 \%$ du potentiel total. Sur ces $490 \mathrm{kTEP}, 247$, soit la moitié, viennent de ressources bien connues, ce qui est économiquement favorable. Le créneau des moyennes profondeurs devrait alors s'ouvrir car les coûts moins élevés en investissements sous-sol permettraient le montage d'opérations avec un nombre unitaire de logements plus faible (de 500 à 1500 selon les cas).

Par ailleurs, concernant les banlieues sud et sud-est, nord et nord-est de Paris, où l'ensemble des projets au Dogger entraîne une saturation à peu près complète de 


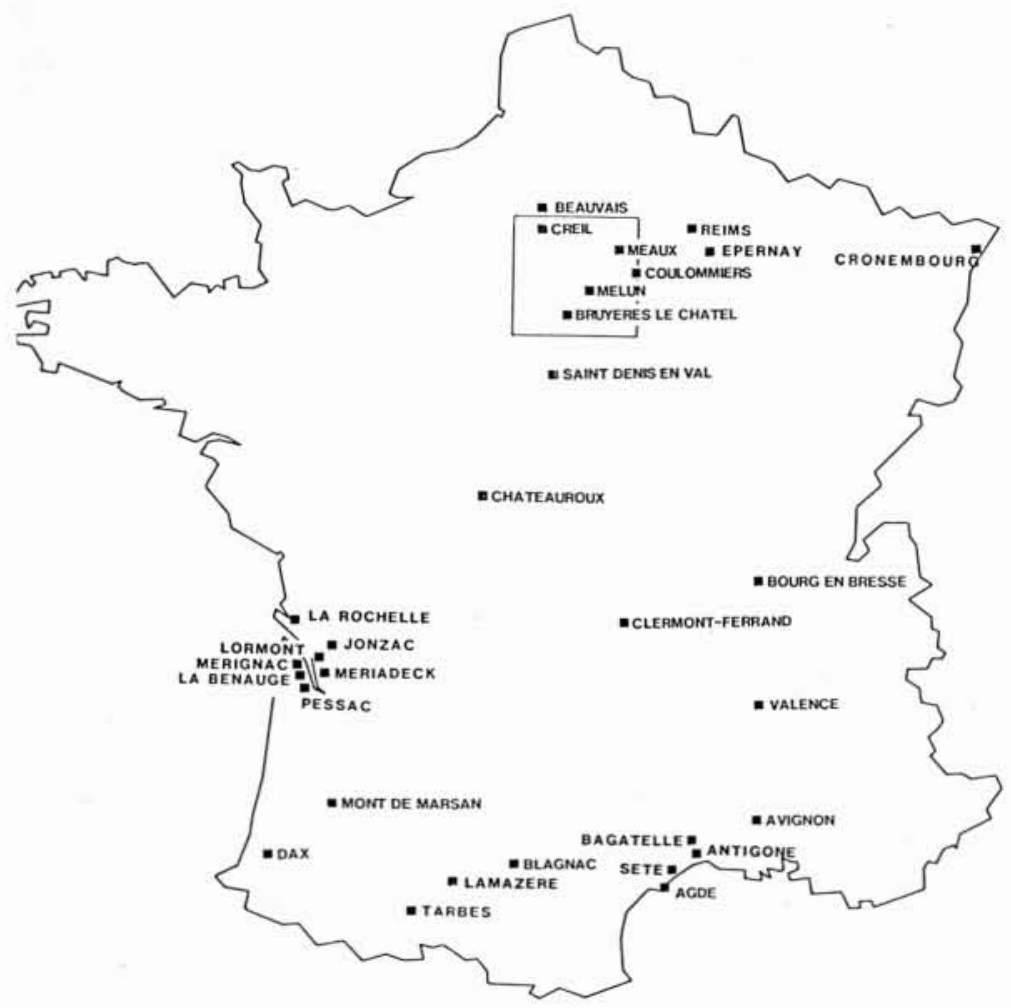

LA GÉOTHERMIE EN RÉGION PARISIENNE

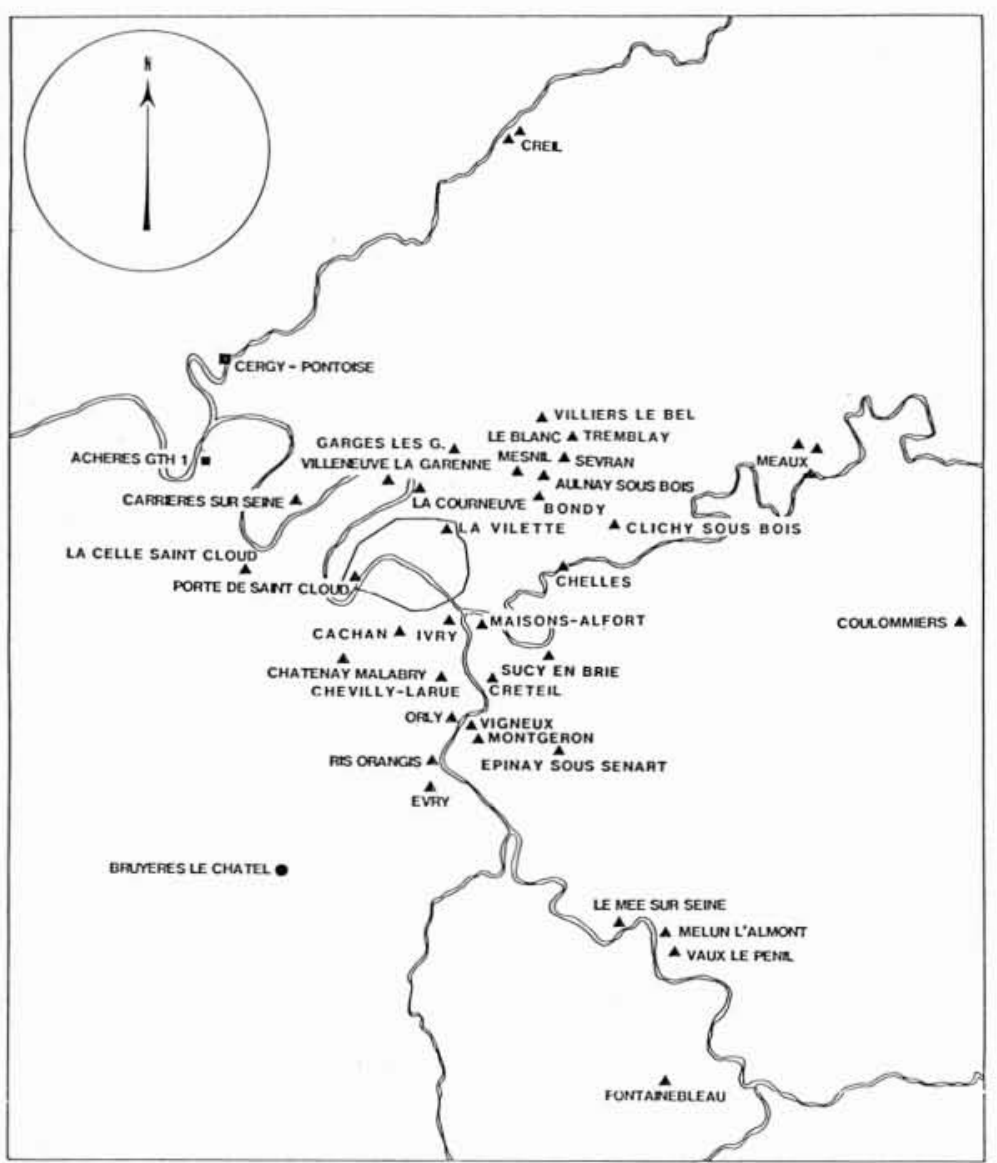

LEGENDE

- Forage géothermique au Trias

A Forage géothermique au Dogger

- Forage géothermique au Barrémie l'espace disponible, le recours au Néocomien, voire au Lusitanien, devrait permettre d'augmenter le nombre d'exploitations.

Pour d'autres régions, telles que l'Aquitaine, la Lorraine, l'Alsace, le Dogger du pourtour du Bassin Parisien, le Languedoc-Roussillon ou le Couloir Rhodanien, il n'est pas impossible que le potentiel des ressources à moyenne profondeur soit supérieur à celui des ressources profondes. Cela concerne alors un ensemble de régions beaucoup plus vaste que précédemment et un nombre accru d'utilisateurs potentiels.

L'utilisation énergétique des ressources à faible profondeur et très basse température constitue également une possibilité non négligeable, car les coûts d'investissement sont encore plus faibles.

Une approche effectuée avec le concours d'EDF montre que, dans le secteur de l'habitat collectif, 12 à 15000 équivalents-logements sont déjà équipés en France avec ce type d'énergie, essentiellement sur nappes peu profondes. L'industrie, l'agriculture (serres) et le tertiaire représenteraient au total 5000 équivalents-logements sur le même type de ressources. A terme, les objectifs du programme PERCHE sont d'équiper $50 \%$ du tertiaire d'hébergement, toutes techniques confondues.

En supposant que les opérations par pompes à chaleur sur aquifères ne représentent que $20 \%$ de ces objectifs, le potentiel réalisable se situera entre 8 et 10000 équivalents-logements par an, soit une substitution de l'ordre de 10000 TEP par an, soit le tiers des résultats attendus des opérations engagées en géothermie profonde en 1984.

Rappelons que les ressources hydrogéologiques françaises dont les nappes ont une température comprise entre 10 et $20^{\circ} \mathrm{C}$ couvrent $30 \%$ de la surface du territoire. L'alimentation et le taux de renouvellement de ces aquifères permettent souvent leur exploitation en puits unique en zone de faible urbanisation, ou selon d'autres dispositifs en zone plus urbanisée.

Peut-on craindre une surexploitation éventuelle ou des conflits d'usage avec les utilisateurs habituels de ces nappes? Les études économiques menées dans le cadre des inventaires départementaux ont montré que, sauf cas exceptionnels, l'exploitation des nappes par pompes à chaleur ne commençait à trouver sa rentabilité que pour des collectifs d'au moins 50 à 100 équivalents-logements. Par ailleurs, les mesures nouvellement adoptées dans le cadre de la procédure AQUAPAC devraient permettre d'assurer une certaine gestion en matière d'exploitation de l'énergie thermique des nappes superficielles.

Enfin, il faut mentionner les actions de recherche menées actuellement en géothermie par plusieurs laboratoires (IMRG, Ecole Nationale Supérieure des Mines/ ARMINES, CNRS/PIRSEM, CEA, IFP), actions auxquelles participent également des sociétés d'ingéniérie ou de recherche pétrolière dans le cadre des opérations en cours de réalisation (GEOTHERMA, BURGEAP, ELF-ERAP/DIENIC).

Les objectifs prioritaires de la recherche sont étroitement liés à l'application : utilisation des nappes phréatiques par pompes à chaleur ainsi que pour le stockage de calories, exploration d'objectifs nouveaux (Lusitanien), exploitation des réservoirs basse température classiques, notamment eaux du Dogger et du Trias, prospection et exploration des gisements à haute température. 
Des objectifs à plus long terme portent sur la production d'électricité à partir de fluides à moyenne température $\left(100-150^{\circ} \mathrm{C}\right)$ au moyen de centrales à fluide binaire. Enfin, dans le cadre d'un programme géothermie profonde généralisée (GPG), l'exploration des roches chaudes sèches en zone de gradient normal est envisagée avec analyse économique préalable de l'utilisation de cette ressource.

En quelques années, la France s'est hissée au premier rang mondial en matière de géothermie basse température. Ce type d'énergie suscite actuellement un grand intérêt dans le monde. Cette activité peut être exportée (ingéniérie, entreprise, équipements).

Le marché de la géothermie basse température est de plus l'élément porteur indispensable à la conquête des marchés de la géothermie haute température qui sont demandeurs d'équipements lourds (turbines, alternateurs, etc.). A ce titre, les travaux en cours dans les départements et territoires d'outre-mer (Guadeloupe, Réunion, Martinique, etc.) constitueront une "vitrine " qui devrait permettre à nos équipes d'émerger sur le marché mondial de la haute température.

CARACTÉRISTIQUES DES PRINCIPALES OPÉRATIONS RÉALISÉES (avril 1983)

\begin{tabular}{|c|c|c|c|c|c|c|c|c|c|c|c|c|}
\hline \multirow{2}{*}{ oersections } & \multirow{2}{*}{$\begin{array}{c}\text { matent } \\
\text { Dourmact }\end{array}$} & \multirow{2}{*}{$\begin{array}{l}\text { Maitat } \\
\text { Oouvanat } \\
\text { Otheout }\end{array}$} & \multirow{2}{*}{ 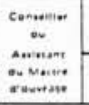 } & \multicolumn{2}{|c|}{$\begin{array}{l}\text { Maitats } \\
\text { Dofuvats }\end{array}$} & \multirow{2}{*}{ 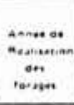 } & \multicolumn{3}{|c|}{ 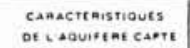 } & \multirow{2}{*}{ 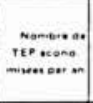 } & \multirow{2}{*}{ 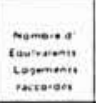 } & \multirow[t]{2}{*}{ oustavdtions } \\
\hline & & & & sovis soc & SUMEACE & & Aatwati: & $\begin{array}{ll}\mathrm{rc} \\
\mathrm{in}\end{array}$ & 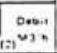 & & & \\
\hline 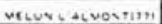 & STANL: & \multicolumn{4}{|c|}{$\cos$} & 199 & oaner & $n$ & 100 & 1500 & 2900 & cantention \\
\hline Sever seose int & $\operatorname{cocu}$ & & & CEOTHEANA & & 1978 & Oesuer & $n$ & 700 & .900 & 3000 & canceweon \\
\hline 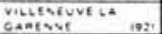 & & & $19 \%$ & Davien & 31 & 50 & 1400 & 1900 & cencenten \\
\hline $\cos 16.160$ & onimiveral & 1 & - & onsu & FIST'́ & 1076 & oower & se & 190 & 3000 & 4200 & \\
\hline magne & urte & \multicolumn{4}{|c|}{ M. cas } & ופיות & foctats & $n$ & 90 & 1000 & zasasinat & concromen \\
\hline 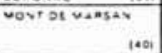 & coctites & \multicolumn{4}{|c|}{ EL coc } & 1911 & Sorencien & $\because$ & 300 & 2000 & 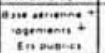 & concenson \\
\hline & w.. & \multicolumn{2}{|c|}{ coc } & SACY & & 1978 & cenomencm & 36 & 190 & 600 & 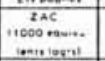 & Cumemingen \\
\hline 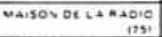 & Oan" & & & & & 1969 & anm & 2 & so & & & \\
\hline $20020 \mathrm{C}$ & va... & & & $\operatorname{Bacu}$ & tacravo & 19000 & Then & 6 & 40 & 450 & & \\
\hline ctaco.ovtaistiast & Eracterer & & & $\sec$ & QETUAE & 1980 & Dossen & 3 & 200 & 2000 & 2000 & 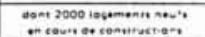 \\
\hline nctotane & oif ctovat & & & oacu & ott & 1980 & Trine & "4 & 120 & $29 \mathrm{co}$ & conditson & 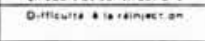 \\
\hline Coviovuefes ont & 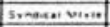 & & Grockentent & $\operatorname{Aag} n$ & secotes & 1980 & Douse & $n$ & 350 & 2000 & 2300 & \\
\hline vontactoon 1911 & 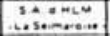 & & coochentert & GCotwenas & auxitec & 19800 & Despe & $n$ & 20 & 2900 & 2100 & 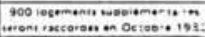 \\
\hline a couaviuve suat & 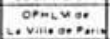 & & Oecosentent & onsu & attunt & 1900 & Dosse & so & 180 & 2000 & 3000 & 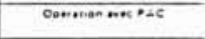 \\
\hline 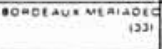 & 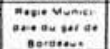 & $\operatorname{san} 0$ & Cosecastout & ancu & TETA & 1980 & comamentien & 53 & 190 & 1600 & 1200 & \\
\hline 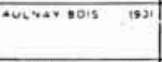 & 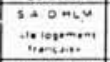 & & Goosnestest & ancu & ero & 1908 & Doser & $"$ & 230 & 3500 & 3100 & 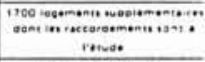 \\
\hline Ocouratis & Drasarout & & seantent & ARSU & Teta & $(981$ & Dosa. & .1 & 100 & 1.00 & 1300 & 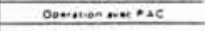 \\
\hline Cuich-ocess 1921 & vili. & Soaceatas & & $\operatorname{sacu}$ & 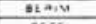 & 1581 & Donat & $n$ & 230 & 27200 & 2300 & \\
\hline ona. & onteduce & & Geacentiont & OtotetanA & sers & 1981 & Doset & 16 & 396 & 2900 & 2100 & \\
\hline 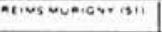 & vate & stoma & Goecroteout & $\operatorname{mon}$ & Tets & 1981 & Luticanien & .7 & $\circ$ & 1050 & 1200 & 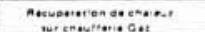 \\
\hline $64021=1 \quad 1321$ & 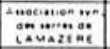 & CAcco. & & ELF & & 1981 & cosent & 56 & 100 & 1500 & 1600 & \\
\hline trent & siteat & crevar & Geacanow & ancM & Fita & 1981 & Dosest: & $"$ & 200 & 3000 & 2500 & 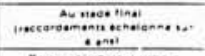 \\
\hline 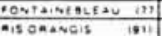 & Songisen min... & GCOCMALTUA & & sacon & of ${ }^{\circ}$ & 1902 & Daser. & 1. & 130 & 1900 & 1000 & 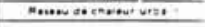 \\
\hline 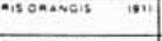 & 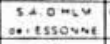 & Geocmative & & Geto:-t:MA & OET & 1962 & Dosest & 72 & 720 & 3100 & 2500 & \\
\hline 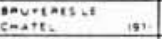 & cet & & & $\theta \times C \infty$ & satist & 1984 & nerocension & 30 & 190 & 1700 & 1000 & 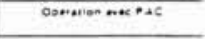 \\
\hline$\theta$ essac & 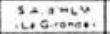 & & 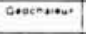 & ancen & $7+4$ & 1932 & conomanien & 4 & 200 & 1450 & 1600 & Dotwetonnacence \\
\hline reture as an inl & sorestat-in. & orocmaliun & & 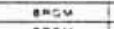 & areated & 1989 & Degist & $\theta$ & 790 & 2000 & 2300 & \\
\hline 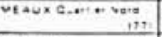 & sonesisen ancot & Grogmatrua & & ancen & Fet & 1992 & Dovert & $"$ & 250 & 3900 & 5000 & 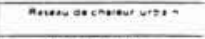 \\
\hline Acutects & 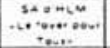 & & Geeent... & cetoratand & oft & 1962 & Dasti: & $"$ & 500 & 2500 & 2100 & 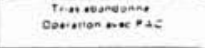 \\
\hline a cousueure ver: & 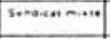 & & cosec.... & $\operatorname{arcu}$ & Ets & 1902 & Dosere & st & 200 & 3000 & 3000 & 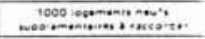 \\
\hline sevesen & SCANAA & $5+65$ & 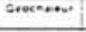 & $\sec$ & AETUEE & 1907 & Doser & $" 0$ & 290 & 2000 & 2000 & 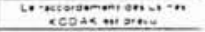 \\
\hline ereavar & vaile & seoven & cosecr.... & $9=00$ & $7 t+2$ & .983 & Onover & 60 & 0 & 2900 & 3000 & \\
\hline $\operatorname{cosectin}$ & $\cos _{\cos } \cos x=$ & & coectenter & $\operatorname{sen} n$ & $76+4$ & 19082 & Doover & 59 & 200 & 2000 & 3200 & 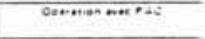 \\
\hline conte of sichoug & vinesenas is & & osecent. & Ceotmenas & Tets & 1902 & 0ooser. & 62 & 250 & 3200 & 3000 & 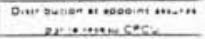 \\
\hline 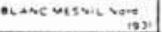 & seaven & s0060et 31 & coucn... & ancen & ocaim & .902 & Dense & ఊ & 230 & 2000 & 2300 & \\
\hline Actithstchoug in & sicke & & $\omega$ & ces & & 1982 & Oone: & 4 & 70 & 1900 & 2350 & Cacr....an cactit: \\
\hline 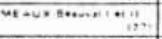 & $5 x+4 \cos -1+0 \mid$ & Getocmateun & & ancu & teta & 1962 & onent & $n$ & 200 & 12500 & 9500 & 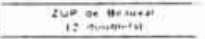 \\
\hline 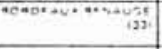 & 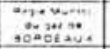 & $\sin =0$ & & $8.05 \%$ & Whresas & 1901 & $\operatorname{conan} x+\cos$ & s & 190 & 1300 & 1,000 & \\
\hline 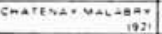 & senticet mint & orocmalfun & & $\operatorname{cotatentan}$ & seavet & 1982 & Dosate & 61 & 220 & 2600 & 2700 & 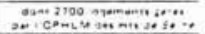 \\
\hline CmattaveOC: 1901 & 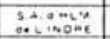 & & $0.00+n+\infty)$ & GEOT-iAGA & & 1583 & Then & 30 & $1: 0$ & 1100 & 1500 & 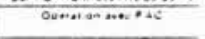 \\
\hline vausctermis int & ving - sen & $\mathrm{ces}$ & & Geormetana & & 1902 & Oane & $n$ & 200 & $32 \% 0$ & 7900 & Cansen... \\
\hline $\begin{array}{l}\text { SA+cet: Las } \\
\text { covesses }\end{array}$ & vin. & satus & sesenten & ancin & otan:s & 1903 & Ooser" & 67 & 230 & 2000 & 2500 & 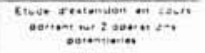 \\
\hline
\end{tabular}

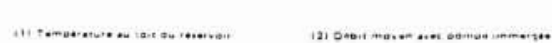


M. GUEDE. - L'objectif fixé en 1977, à savoir 1 million de TEP géothermiques, émanait-il d'un optimisme scientifique et politique ? Existe-t-il actuellement des subventions pour la géothermie?

M. VATHAIRE répond que l'objectif gouvernemental de 1977 était en effet de 1 million de TEP géothermique à constituer en 1990. Aujourd'hui nous en sommes à 180000 TEP. Deux aspects sont à considérer :

1) on estime à l'heure actuelle le gisement français à 600000 TEP;

2) les collectivités locales font appel aux Pouvoirs Publics qui prennent en charge le risque minier par le biais de subventions. Depuis 1982 les financements étaient pris en charge par la deuxième tranche du fonds spécial "grands travaux ". Ils sont devenus inexistants à partir de la troisième tranche : on a considéré que l'énergie n'était plus un secteur prioritaire.

Les industries géothermiques françaises représentent à l'heure actuelle 3500 personnes et plus de 45 sociétés, ainsi qu'un savoir-faire qui va disparaître. C'est d'autant plus navrant que nous avions quasiment acquis un leader ship sur le plan mondial en matière de développement de ce type d'énergie et que nous commencions déjà à l'exporter. Demain, nous allons probablement perdre tous ces acquis.

M. CLÉMENT demande si des études de marché relatives à la géothermie de très faible profondeur $(500 \mathrm{~m})$ ont été menées.
LE PRÉSIDENT répond que des inventaires départementaux et des études de pré-faisabilité ont été faits. Il a été montré, par exemple, que l'utilisation d'une nappe phréatique située à $30 \mathrm{~m}$ de profondeur devient économiquement très intéressante à partir d'un ensemble collectif de l'ordre de 50 logements. D'autre part, concernant les émetteurs à basse température, une opération pilote a été conduite en région Centre. II s'agit d'un ensemble H.L.M. de 100 logements où, en trois ans, les dépenses de chauffage, investissements compris, ont été divisées par trois.

M. GRJEBINE pose le problème de la rentabilité des opérations de géothermie et remarque que ces dernières font l'objet de calculs d'amortissement linéaire sur 7 ans et demi alors que les opérations utilisant d'autres sources d'énergie sont indexées sur l'inflation. II conclut que la géothermie, si une publicité plus importante lui était faite pourrait se développer beaucoup plus.

M. VATHAIRE rappelle que la géothermie n'a pas la puissance politique des autres secteurs de production même si elle concerne en France 45 Sociétés, 3500 personnes et représente un chiffre d'affaires de l'ordre de 1 milliard de francs par an. De ce fait, l'impact commercial de la géothermie est tout à fait relatif et la position de Gaz de France, par exemple, ne facilite pas son expansion. Il regrette enfin l'absence de plans énergétiques régionaux.

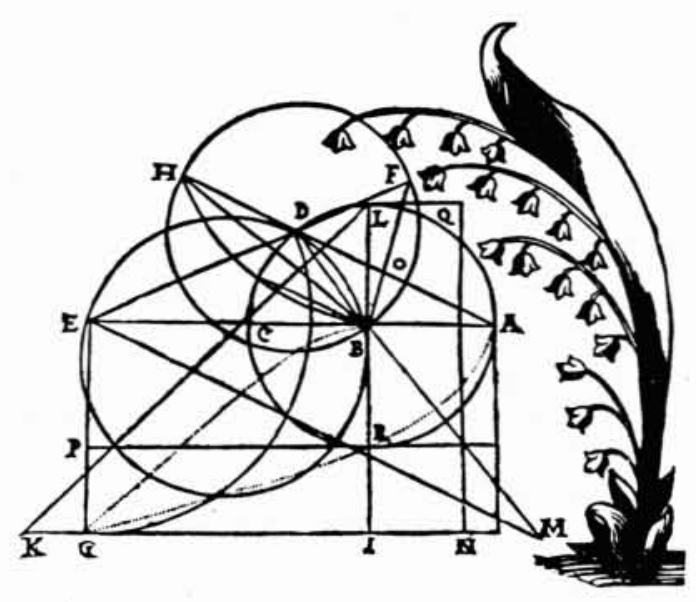

